\title{
Development and Evaluation of Patient Information Leaflet for Diabetic Foot Ulcer Patients
}

\author{
Sonal Sekhar M, ${ }^{1,}{ }^{*}$ Unnikrishnan MK, ${ }^{1}$ Navya Vyas, ${ }^{2}$ and Gabriel Sunil Rodrigues ${ }^{3}$ \\ ${ }^{1}$ Department of Pharmacy Practice, Manipal College of Pharmaceutical Sciences, Manipal University, Manipal, India \\ ${ }^{2}$ Department of Public Health, Manipal University, Manipal, India \\ ${ }^{3}$ Department of Surgery, Kasturba Medical College Hospital, Manipal University, Manipal, India \\ "Corresponding author: Sonal Sekhar M, Assistant Professor-Selection Grade, Department of Pharmacy Practice, Manipal College of Pharmaceutical Sciences, Manipal \\ University, Manipal, India-576104. Tel: +91-8202922482, Fax: +91-8202571998, E-mail: sonalsekhar@gmail.com
}

Received 2016 October 02; Revised 2017 April 10; Accepted 2017 May 24.

\begin{abstract}
Background: Patient education (PE) is as important as medical and surgical interventions in the management of diabetic foot ulcer (DFU). Patient information leaflets (PILs) are globally accepted patient counseling aids.

Objectives: This study aimed at developing PILs for DFU patients and investigating its validation.

Methods: The PILs were prepared based on different model leaflets available from various online resources, including "Patient UK". The PILs readability was evaluated by Flesch/ Flesch-Kincaid readability (FRE/FK-GL) method before user-testing ( $\mathrm{n}=34 \mathrm{DFU}$ patients) by quasi-experimental methods in patients with DFU. Additionally, user-opinion on legibility and content of the PIL was also determined. Baker Able Leaflet Design (BALD) method was employed to assess the layout and design characteristics of the PIL.

Results: The best FRE score achieved was 73.9 and the FK-GL score was 6.1. The mean BALD assessment score for English and Kannada versions of PIL were 27 and 26, respectively. The ICC of the test-retest reliability of user-testing and user-opinion questionnaires in both English and Kannada ranged from 0.91 to 0.96 . The overall user-testing knowledge-based mean score significantly improved from 43.4 to $69.7(\mathrm{P}<0.05)$. Overall, $82.4 \%$ of patients reported overall user-opinion on legibility and content of the PIL as good.

Conclusions: The developed PILs met the criteria of fairly easy readability and good layout design. The user-opinion of the majority of patients reported the PIL content, legibility, and design as good. The Pictogram-based PILs (P-PILs) was found to be an effective PE tool in DFU patients.
\end{abstract}

Keywords: Diabetic Foot Ulcer, Patient-Education, Patient Information Leaflets

\section{Background}

Patient education (PE) is a central component in the self management of diabetes complications in both prevention and treatment (1-3). It is one of the major management strategies for prevention and treatment of diabetic foot ulcer (DFU). Patient involvement is vital for the successful care of DFUs. The primary task of the health care team is to boost patient's self confidence, improve patient awareness and provide support. Usually, patients and their families provide $95 \%$ of the care needed to manage diabetic complications (4).

Poor knowledge of foot care practices is an important risk factor for foot problems among diabetics. Studies have demonstrated deficiencies in knowledge and practices regarding foot care, particularly among rural populations with diabetes (5). Recent Cochrane reviews found that foot care knowledge and self-reported patient behavior appear to be positively influenced by education $(6,7)$.

Patient information leaflets (PILs) are globally accepted instruments to educate patients regarding diseases, medications, and lifestyle modifications (8).
Pictogram-based PILs (P-PILs) are the advanced version of PILs (9-11). When designing PILs, the most important elements to be considered are literacy and the individual's comprehension level. Good readability, layout, and design are important factors for developing PILs (12-14).

User-testing is intended to assess the readability of a document by a group of selected test subjects (13). Usertesting ensures that the information is legible, clear, and easy to use, hence patients could locate and understand vital information within the PILs (13, 15-17). An internationally accepted Baker able leaflet design (BALD) criteria was employed for good design characteristics of PILs. A well-designed PIL with good readability scores assists patients to comprehend the content of PILs that may in turn improve their knowledge, attitude and practices (KAP) towards disease management (18).

\section{Objectives}

This study aimed at preparing and investigating the validation of PILs and performing readability testing of PIL for DFU patients. 


\section{Methods}

\subsection{Preparation of Patient Information Leaflet}

The PIL was prepared by referring different model leaflets, which were available from various online sources such as "Patient UK", www.diabeticfoot.org.uk, and www.diabetes-healthnet.ac.uk (19-21). "Diabetes in Scotland" website was used to compare different versions of published leaflets (22). Information was also gathered from resources, such as "Micromedex", "Up-to-date", "Medline", and "Medscape" database (23). Content of the PIL included advice on daily care, dressings, footwear, and treatment of DFUs. The PIL was prepared based on the standard guidelines $(10,15)$. The content, design, and pictograms of the PIL were validated by an expert committee consisting of a physician, a surgeon, and 3 academic pharmacists. The changes were made in the PIL as per the expert committee's directions for content validation. The BALD method was employed to assess the layout and design characteristics of PIL. The validated English version of PIL (Figure 1) was translated to Kannada, the local language, by a language expert. The process of translation included 3 steps; forward translation, backward translation, and finally patient-testing.

\subsection{Assessment of Readability of Patient Information Leaflet}

Readability of the developed PIL was evaluated by 2 method readability formula, Flesch reading Ease/FleschKincaid grade level (FRE/FK-GL) and user-testing (10, 15, 24, 25).

\subsubsection{Flesch/Flesch-Kincaid Readability Test}

The study used an adult literacy readability calculator, known as the 'FRE' and the 'FK-GL' to estimate the ability required to read the PIL, using a formula based on sentence length and frequency of polysyllable words in the content. The FRE score rates texts on a 0 to 100 point scale. A higher score indicates that the document is easier to read and understand (24).

The PIL was also reviewed for simple measure of Gobbledygook (SMOG) grade, which estimates the level of education needed to understand the text (26). Readability of the PIL was calculated using the website www.readabilityscore.com (25).

\subsubsection{User-Testing Readability Test}

Readability of the PIL was also assessed by the usertesting method (10). A user-testing questionnaire, both English and Kannada, consisting of 10 closed-end (Yes/No) questions, was prepared based on the content of the PIL. The questionnaire was validated for face validation before assessing the knowledge of the patients. During usertesting, the questionnaire was administered to the DFU patients for assessing baseline knowledge. Next, PIL of either English or Kannada version was given to the patients, based on their choice, and they were allowed to read the PIL for 20 minutes. Next, the patients were again administered the user-testing questionnaire to re-assess the knowledge. At the end, the response was evaluated using the following formula.

$\begin{aligned} \text { Response evaluation }= & \frac{\text { Total number of correct responses }}{\text { Total number of actual responses }} \\ & \times 100\end{aligned}$

After knowledge assessment by user-testing, a questionnaire was used in both English and Kannada to assess user-opinion regarding the PIL, on content, layout, and design. The questionnaire consisted of 4 questions with a score ranging from 1 to 5 . The interpretation of the final scores is given below. User-opinion questionnaire was validated by the expert committee in a similar fashion. Testretest reliability of the user-testing and user-opinion questionnaires were assessed with a sub-sample of 20 randomly selected DFU patients, for which, Kannada and English versions of both questionnaires were administered twice, a week apart.

The interpretation of the user-opinion scores was as follows:

Good: "16 - 20" score

Average: "10 - 15 " score

Poor: “<10" score

\subsection{Study Design and Subjects}

A quasi-experimental pre-and post-design evaluation of PILs within the intervention group (IG) without a control group (CG) was carried out by 34 DFU patients with prior approval of institutional ethical clearance obtained from Kasturba hospital (KH), Manipal, India (IEC 143/2012). The inclusion criterion of the study was recent diagnosis of DFU and provision of a written informed consent. Sociodemographics, such as age, gender, and educational status of the patients were collected, in addition to economic status.

\subsection{Statistical Analysis}

The intra-class correlation coefficients (ICC) were computed to assess the test-retest reliability of both usertesting and user-opinion questionnaires. The ICC values $\geq$ $0.9,0.7$ to 0.89 , and $<0.7$ were considered as highly reliable, moderately reliable, and weakly reliable, respectively. Descriptive statistics were used to summarize the data. The user-testing scores, both baseline and after 20 minutes, 


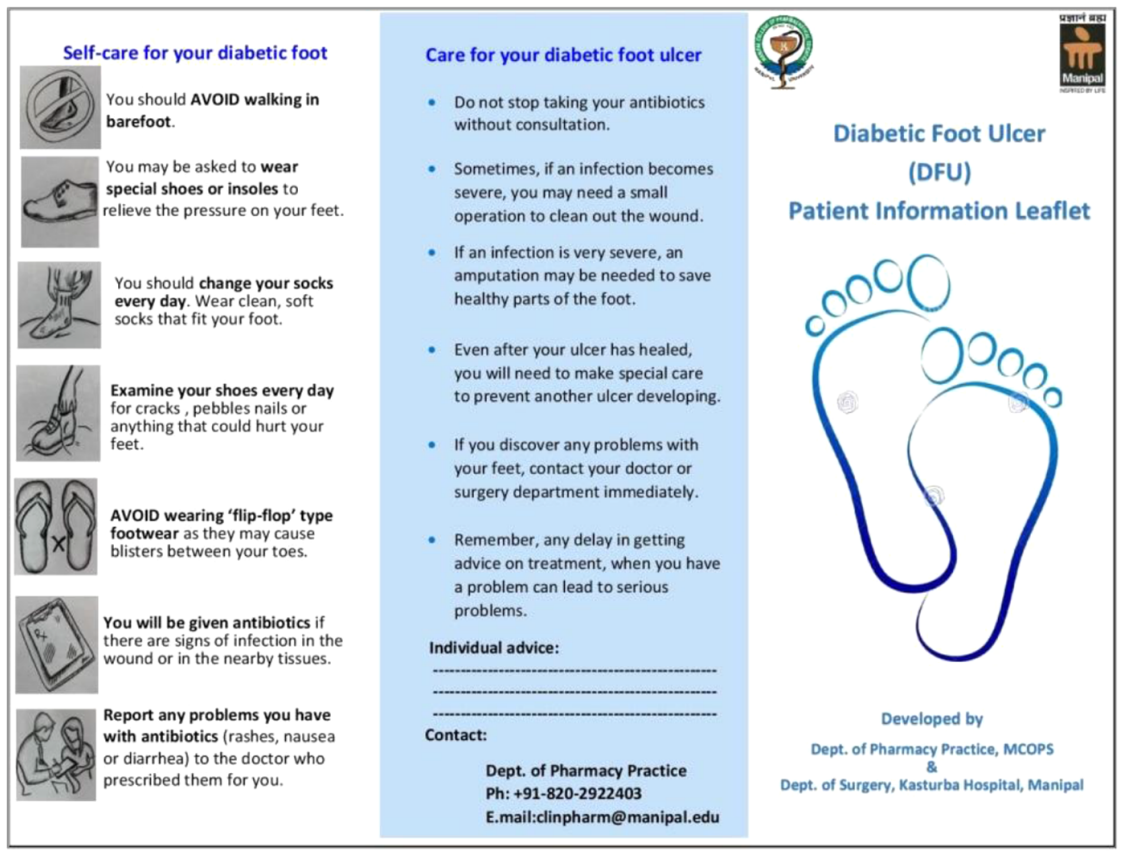

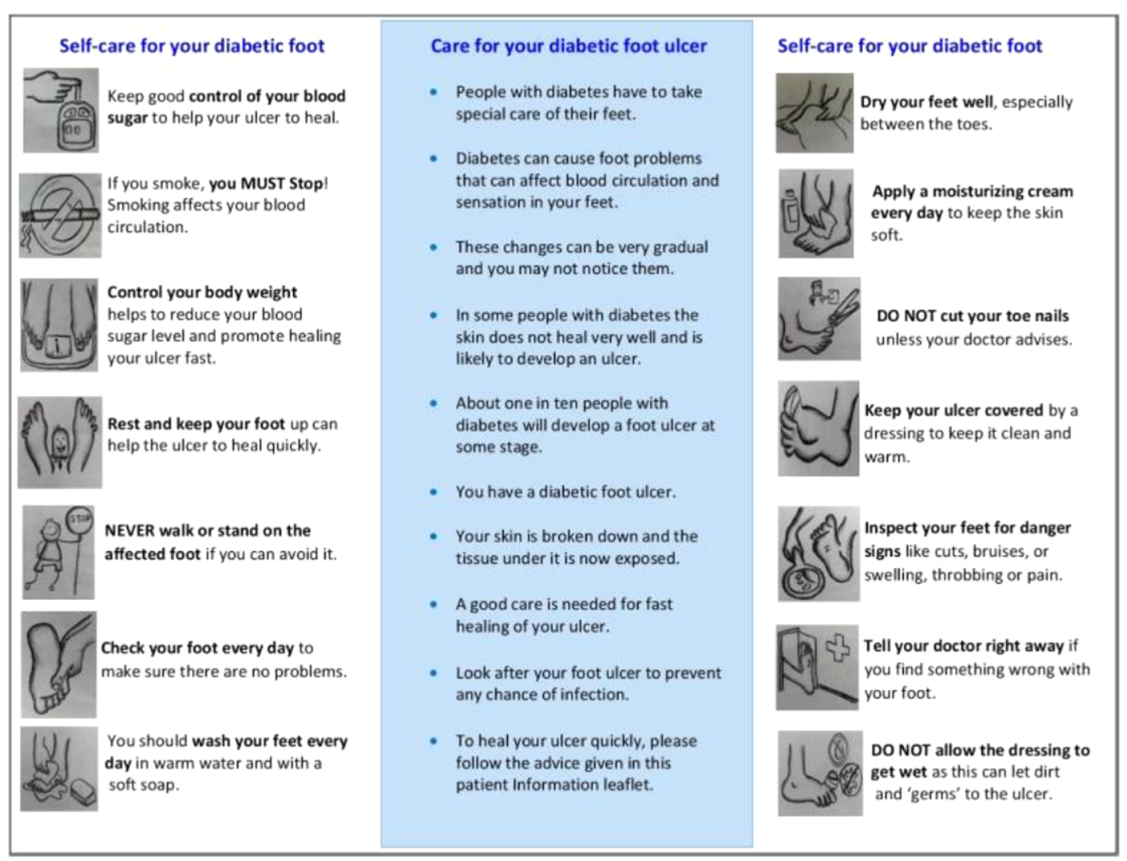

Figure 1. Patient Information Leaflet for DFU (English)

were compared using paired $\mathrm{t}$ test. P values $<0.05$ were considered statistically significant. Data were entered and analyzed using SPSS version 20.0 software.

\subsection{Sample Size}

The minimum sample size of the study was calculated as 33, based on the change in patient's knowledge of usertesting scores from baseline to post-intervention score. Sample size was calculated using nMaster software with 
the following formula.

$n=\frac{\left(Z_{1-\frac{\alpha}{2}}+Z_{1-\beta}\right)^{2} \sigma^{2}}{d^{2}}+2$

Where,

Mean of the two standard deviations $(\sigma)=10$

$\mathrm{Z}_{1-\alpha / 2}$ for $\alpha(5 \%)=1.96$

$\mathrm{Z}_{1-\beta}$ for $80 \%$ power $=0.84$

5)

Minimum significant difference in the two groups $(\mathrm{d}=$

\section{Results}

\subsection{FRE/FK-GL Readability Test Score}

During the development of PIL, readability was assessed using an online readability calculator. Readability of the PIL was reassessed after periodic modification, demonstrating steady improvement in readability scores after each modification. The best FRE score and FK-GL scores were 73.9 and 6.1, respectively (Table 1).

Table 1. PIL Readability Tests Score (Final Version)

\begin{tabular}{lc}
\hline Readability Formula & Grade \\
\hline Flesch-Kincaid grade level & 6.1 \\
Gunning-Fog score & 8.5 \\
\hline Coleman-Liau Index & 10.5 \\
\hline SMOG index & 6.3 \\
\hline Automated readability index & 5.9 \\
\hline Average grade level & 7.5 \\
\hline
\end{tabular}

Abbreviation: SMOG: Simple Measure of Gobbledygook.

\subsection{Leaflet Design}

The mean BALD assessment score for English version and Kannada version of PIL were 27 and 26, respectively.

4.3 User-testing readability study

\subsubsection{Demographics of Study Subjects for User-Testing and User-} Opinion of PIL

Thirty-four newly diagnosed patients with DFU were included in the user-testing. Demographic details of the patients are shown in Table 2.

\subsubsection{User-Testing and User-Opinion Scores of the PIL}

Out of 34 patients, 20 (58.8\%) preferred Kannada PILs and 14 (41.2\%) preferred English PIL. The overall pre and post user-test on knowledge assessment mean scores of the PIL significantly improved from 43.4 to 69.7 (Table 3). Overall user-opinion on legibility and content of the PIL was reported as good by $82.4 \%$ (Table 4 ).

\begin{tabular}{|c|c|}
\hline Parameters & Values \\
\hline $\operatorname{Age}($ Mean $\pm S D), y$ & $58.3 \pm 7.4$ \\
\hline \multicolumn{2}{|l|}{ Gender } \\
\hline Male & $26(76.5)$ \\
\hline Female & $08(23.5)$ \\
\hline \multicolumn{2}{|l|}{ Educational status } \\
\hline Primary school & $06(17.7)$ \\
\hline Middle school & $08(23.5)$ \\
\hline High school & $14(41.2)$ \\
\hline Intermediate & $03(8.8)$ \\
\hline Graduate & $03(8.8)$ \\
\hline \multicolumn{2}{|l|}{ Socio-economic status } \\
\hline Upper & $02(5.9)$ \\
\hline Upper middle & $05(14.7)$ \\
\hline Lower middle & $12(35.3)$ \\
\hline Upper lower & $08(23.5)$ \\
\hline Lower & $07(20.6)$ \\
\hline
\end{tabular}

Abbreviation: SD, Standard Deviation.

${ }^{\mathrm{a}}$ Values are expressed as No. (\%).

4.2.3. Test-Retest Reliability of User-Testing and User-Opinion Questionnaire

Out of 20 patients with DFU, 12 were assessed for Kannada and the remaining 8 were assessed for the English versions of the questionnaires. The ICC reliability for both versions of the questionnaire ranged from 0.91 to 0.96 (Table 5).

\section{Discussion}

This study was the first of its kind in India that developed a PIL for patients with DFU and was evaluated for both readability and user-testing. The only previous study in India, conducted on PIL for DFU, was "Preparation and readability assessment of patient information leaflets for diabetic foot ulcers" by Roy et al., in which the readability formula method was employed (16).

If the FRE score of a PIL is $<60$, the text is considered to be difficult to read by the general public. In this study, mean readability scores of the PIL were found to be 73.9. These scores suggest that the readability of the PIL is "fairly easy". The higher the readability, the better is the acceptance of the PILs by the patients (15).

The FK-GL gives a score equivalent to a school grade level. This score can be converted to a reading age by 
Table 3. User-Testing Scores of Patient Information Leaflets ${ }^{a}$

\begin{tabular}{lcccc}
\hline Type of PIL Users & Pre-test Score & Post-test Score & Mean Difference \pm SD & PValue \\
\hline English $(\mathbf{n}=\mathbf{1 4})$ & $45.8 \pm 10.6$ & $74.3 \pm 11.5$ & $28.5 \pm 11.1$ & 0.0001 \\
Kannada $(\mathbf{n}=\mathbf{2 0})$ & $41.1 \pm 11.4$ & $65.1 \pm 10.9$ & $24.0 \pm 9.8$ & $26.3 \pm 10.5$ \\
Overall $(\mathbf{n}=\mathbf{3 4})$ & $43.4 \pm 9.5$ & $69.7 \pm 11.3$ & & \\
\hline
\end{tabular}

${ }^{\mathrm{a}}$ Data are presented as mean \pm SD.

Abbreviation: SD, Standard Deviation; PIL, Patient Information Leaflet.

Table 4. User-Opinion Scores of Patient Information Leaflets ${ }^{\mathrm{a}}$

\begin{tabular}{|cc|}
\hline User-Opinion Category & No. of Patients \\
\hline English & $11(78.6)$ \\
\hline Good & $03(21.4)$ \\
\hline Average & - \\
\hline Poor & \\
\hline Kannada & $17(85.0)$ \\
\hline Good & $03(15.0)$ \\
\hline Average & - \\
\hline Poor & \\
\hline Overall & $28(82.4)$ \\
\hline Good & $06(17.6)$ \\
\hline Average & - \\
\hline Poor & \\
\hline
\end{tabular}

${ }^{\mathrm{a}}$ Values are expressed as No. (\%).

adding 5. The FK-GL of the PIL gave a score of 6.1, which approximately equates to a reading age of between 11 and 12 years old. A previous study on readability of PIL for DFU reported a FK-GL score of 7.1 (16). Gunning-Fog index estimates the years of formal education needed to understand written text on a first reading. It is usually used to confirm whether the text could be read easily by the intended audience $(24,25)$. Gunning-Fog score of the PIL was 8.5, indicating that the text was readable and understandable. Coleman-Liau index of the text of PIL was 10.5, which means the PIL was at a grade level of 10.5, or roughly appropriate for a $10^{\text {th }}$ to $11^{\text {th }}$ grade $(24,25)$. A SMOG index of 6.3 is equivalent to $6^{\text {th }}$ or $7^{\text {th }}$ grade reading level. The SMOG index readability formula measures the years of education a person needs to fully understand a written text(26). A similar SMOG index was reported in a previous study (16). Automated Readability Index(ARI), which rates the understandability of the text, was 5.9, which equates to upper primary school level ( $6^{\text {th }}$ grade), age 11 to 12 years $(16,24,25)$.

A grade level is equivalent to the number of years of ed- ucation a person has had. The average grade level of the text was 7.5, against the value of 10 to 12, expected from a person, who has completed high school in India. Text to be read by the general public in western countries is generally aimed at a grade level of $\leq 8$ (25). Therefore, it could be concluded that these values may be applied meaningfully in the Indian context.

As per the BALD criteria, a PIL scoring $>20$ (Total score is 32) is considered as "good" in layout and design characteristics (18). The PIL developed in this study scored $>25$ in both English and Kannada versions and were similar to an English PIL prepared by the authors in a previous study (16).

In this study, user-testing was employed along with the FRE/FK-GL method to assess the PIL readability. Usertesting is more reliable than the assessment by the formula method because PILs in any language could be tested $(10,15)$. Unlike previous studies, the authors also evaluated user-opinion in addition to user-testing of the PIL (17). It was found that $82.4 \%$ gave a good rating to content, legibility, and design of the PILs. The ICC value of the user-testing and user-opinion questionnaires was $>0.9$ and considered as highly reliable. The study proved that the post-intervention user-testing scores significantly improved from 43.4 to 69.7 ( $\mathrm{P}<0.05$ ) with the use of PILs. Similarly, other studies performed on the impact of PILs in recalling the information provided, in various conditions such as cancer, smoking, renal failure, asthma, diabetes, hypertension, peptic ulcer, and angina showed a significant effect $(11,18)$.

The main strengths of the study were adequate number of participants and response rate to the questionnaires was high. Against the minimum requirement of 20 patients, the authors performed the user-tests on the basis of one-to-one, face-to-face, structured sets of interviews in 34 patients with DFU (10).

\subsection{Conclusion}

The study demonstrated that the PILs were wellreceived by the patients with DFU, also indicating significant improvement in patient knowledge level after read- 
Table 5. Test Re-Test Reliability of User-Testing and User-Opinion Questionnaires ${ }^{\mathrm{a}}$

\begin{tabular}{lccc}
\hline Type of Questionnaire & Test 1(Day 1) Score & Test 2 (Day 7) Score & ICC Value \\
\hline User testing English $(\mathbf{n}=\mathbf{8})$ & $44.6 \pm 11.3$ & $44.2 \pm 10.1$ & 0.91 \\
User testing Kannada $(\mathbf{n}=\mathbf{1 2})$ & $43.2 \pm 12.4$ & $42.9 \pm 13.2$ & 0.96 \\
User opinion English $(\mathbf{n}=\mathbf{8})$ & $17.2 \pm 4.4$ & $17.5 \pm 3.1$ & 0.92 \\
User opinion Kannada $(\mathbf{n}=\mathbf{1 2})$ & $16.4 \pm 8.4$ & $16.8 \pm 5.6$ & 0.93 \\
\hline
\end{tabular}

Abbreviations: SD, Standard Deviation; ICC, Intra-Class Correlation Coefficient.

${ }^{\mathrm{a}}$ Values are expressed as mean $\pm \mathrm{SD}$.

ing the PILs. The PILs met the standards of fairly easy readability and good layout design criteria required for PE. The reading age required to understand the PIL was appropriate for the target group. The user-opinion reported that the majority of the patients rated the PILs' content, legibility, and design as good. The study concluded that pharmacistled PE with PILs had a significant positive impact on the patient's knowledge on DFU.

\section{Acknowledgments}

The authors are thankful to Mr. Jefry Jose, Pharm.D (Intern) for drawing pictograms for the PIL.

\section{Footnotes}

Authors' Contribution: Dr. Sonal Sekhar M developed the study concept and design, acquisition of data, wrote the manuscript, and was the guarantor. Dr. Unnikrishnan MKand Dr. Gabriel Sunil Rodrigues contributed to the critical revision of the manuscript and supervision of the study. Dr. Navya Vyas contributed to the statistical analysis and interpretation of data and drafting of the manuscript.

\section{Conflict of Interests: None.}

Funding/Support: Nil.

\section{References}

1. Soundarya M, Asha A, Mohan V. Role of a diabetes educator in the management of diabetes. Int J Diabetes Dev Ctries. 2004;24:65-8.

2. Malone JM, Snyder M, Anderson G, Bernhard VM, Holloway GA Bunt TJ. Prevention of amputation by diabetic education. Am J Surg. 1989;158(6):520-4. doi: 10.1016/0002-9610(89)90183-9.

3. Chiwanga FS, Njelekela MA. Diabetic foot: prevalence, knowledge, and foot self-care practices among diabetic patients in Dar es Salaam, Tanzania - a cross-sectional study. J Foot Ankle Res. 2015;8:20. doi: 10.1186/s13047-015-0080-y. [PubMed: 26064190].

4. Palaian S, Chhetri AK, Prabhu M, Surulivelrajan M, Ravi Shankar P. Role of pharmacist in counseling diabetes patients. J Pharmacol. 2005;4(1):1-13.

5. George H, Rakesh P, Krishna M, Alex R, Abraham VJ, George K, et al. Foot care knowledge and practices and the prevalence of peripheral neuropathy among people with diabetes attending a secondary care rural hospital in southern India. J Family Med Prim Care. 2013;2(1):2732. doi: 10.4103/2249-4863.109938. [PubMed: 24479039].
6. Dorresteijn JA, Kriegsman DM, Assendelft WJ, Valk GD. Patient education for preventing diabetic foot ulceration. Cochrane Database Syst Rev. 2014(12):CD001488. doi: 10.1002/14651858.CD001488.pub5. [PubMed: 25514250].

7. Valk GD, Kriegsman DM, Assendelft WJ. Patient education for preventing diabetic foot ulceration. A systematic review. Endocrinol Metab Clin North Am. 2002;31(3):633-58. doi:10.1016/S0889-8529(02)00021-X. [PubMed: 12227125].

8. Newton L, Newton D, Clark J, Kenny T, Moseley D, Purves I, et al. Patient information leaflets: Producing understandable PILs. J Inform Sci. 1998;24(3):167-81. doi: 10.1177/0165551984232162.

9. Mansoor LE, Dowse R. Effect of pictograms on readability of patient information materials. Ann Pharmacother. 2003;37(7-8):1003-9. doi: 10.1345/aph.1C449. [PubMed: 12841808].

10. Enterprise and industry directorate-General. Guidelines on the readability of the labelling and package leaflet of medicinal products for human use. Brussels: European Commission 2009. [cited Jan]. Available from: http://ec.europa.eu/health/files/eudralex/vol2/c/2009_ 01_12_readability_guideline_final_en.pdf.

11. Mateti UV, Nagappa AN, Attur RP, Bairy M, Nagaraju SP, Mallayasamy $S$, et al. Preparation, validation and user-testing of pictogram-based patient information leaflets for hemodialysis patients. Saudi Pharm J. 2015;23(6):621-5. doi: 10.1016/j.jsps.2015.01.022. [PubMed: 26702256].

12. Mumford ME. A descriptive study of the readability of patient information leaflets designed by nurses. J Adv Nurs. 1997;26(5):985-91. doi: 10.1046/j.1365-2648.1997.00455.x. [PubMed: 9372404].

13. Murphy J, Gamble G, Sharpe N. Readability of subject information leaflets for medical research. N Z Med J. 1994;107(991):509-10. [PubMed: 7830982].

14. Aldridge MD. Writing and designing readable patient education materials. Nephrol Nurs J. 2004;31(4):373-7. [PubMed:15453229].

15. Renuka P, Pushpanjali K. leaflet preparation and Validation procedures. Univers J Public Health. 2013;1(3):110-4. doi: 10.13189/ujph.2013.010310.

16. Sekhar MS, Rajesh V, Rodrigues GS, Roy RT. Preparation and readability assessment of patient information leaflets for diabetic foot ulcers. J Soc Health Diabetes. 2013;1(2):79-81. doi:10.4103/2321-0656.115304.

17. Smith H, Gooding S, Brown R, Frew A. Evaluation of readability and accuracy of information leaflets in general practice for patients with asthma. BMJ. 1998;317(7153):264-5. [PubMed: 9677221].

18. Adepu R, Swamy MK. Development and Evaluation of Patient Information Leaflets (PIL) Usefulness. Indian J Pharm Sci. 2012;74(2):174-8. doi: 10.4103/0250-474X.103857. [PubMed: 23326002].

19. Diabetes, foot Care and foot ulcers. Patient UK [cited Jan]. Available from: http://patient.info/health/diabetes-foot-care-and-foot-ulcers.

20. Step by Step Guide to Healthy Feet- For people with Diabetes. The diabetic foot: a resource for health care professionals [cited Jan]. Available from: http://www.diabeticfoot.org.uk/. 
21. You and your foot ulcer. A general guide to help you care for yourself and your foot ulcer. Primary Care Trust Manchester Community Health: Manchester NHS; [cited Jan]. Available from: http://www.uhsm.nhs.uk/patients/Diabetes\%20patient\%20leaflets/ Foot\%20Ulcer.pdf.

22. Publications (leaflets). Diabetes in Scotland [cited Jan]. Available from: http://www.diabetesinscotland.org.uk/Publications.aspx? catId=2.

23. Mc Culloch DK. Patient information: Foot care in diabetes mellitus (beyond the basics) [cited Jan]. Available from: http://www.uptodate. com/contents/foot-care-indiabetes_mellitus_beyond_thebasics.

24. Flesch R, Kincaid JP. Flesch-Kincaid grade level readability test [cited Jan]. Available from: http://www.readabilityformulas.com/fleschgrade-levelreadability-formula.php.

25. Readability score [cited Jan]. Available from: https://readabilityscore.com.

26. McLaughlin GH. SMOG grading - a new readability formula.J Reading. 1969;12(8):639-46. 